\title{
Exame de papanicolaou: conhecimentos de mulheres em uma unidade de saúde escola da Amazônia ocidental
}

\author{
Papanicolaou exam: knowledge of women in a school health unit of the western Amazônia \\ Examen de papanicolau: conocimiento de mujeres en una unidad de salud escolar de la \\ Amazonia occidental
}

Josiel Neves da Silva ${ }^{1 *}$, Anitha de Cássia Ribeiro da Silva ${ }^{1}$, Breno de Oliveira Chagas Barreto', Arlindo Gonzaga Branco Junior ${ }^{1,2}$, Claudete Martins de Lima ${ }^{1}$, Rita de Cassia Alves Ferreira Silva ${ }^{1}$.

\section{RESUMO}

Objetivo: Avaliar o conhecimento de mulheres sobre o exame de Papanicolaou em um Centro de Atenção a Saúde da Mulher Método: O estudo foi de caráter descritivo exploratório e de abordagem quantitativa. Para isso, foi aplicado um questionário com perguntas gerais e específicas, após assinatura do Termo de Consentimento Livre e Esclarecido, que avaliou dentre outros fatores, o perfil socioeconômico, escolaridade e o nível de conhecimento sobre o exame. Resultados: Participaram do estudo 100 mulheres, em que, 40\% das mulheres deixaram claro a relação da finalidade/prevenção do câncer do colo de útero. Mais da metade das mulheres responderam saber os requisitos para a realização do exame. Apesar de $5 \%$ das pessoas terem dito que o intervalo entre dois exames depende, as restantes responderam conforme sua rotina ao ambulatório. Quanto ao que deveria ser feito após o resultado, mais de $80 \%$ disseram levar ao médico como condição determinante. Conclusão: As descrições mostraram que parte tem conhecimento sobre o exame de rastreio, mesmo não sendo possível quantificar com exatidão o conhecimento de cada paciente. Os profissionais de saúde exercem papéis fundamentais para fins de informação da saúde em geral, porém, ainda é necessária uma atenção maior na promoção da saúde da mulher.

Palavras-chave: Papanicolaou, Conhecimento, Prevenção do câncer de colo do útero.

\begin{abstract}
Objective: To evaluate the knowledge of women about the Pap smear in a Women's Health Care Center. Method: The study was descriptive and exploratory with a quantitative approach. For this, a questionnaire was applied with general and specific questions, after signing the Free and Informed Consent Term, which assessed, among other factors, the socioeconomic profile, education and the level of knowledge about the exam. Results: 100 women participated in the study, in which $40 \%$ of women made clear the relationship between the purpose / prevention of cervical cancer. More than half of the women answered that they knew the requirements for the exam. Although $5 \%$ of people said that the interval between two tests depends, the rest responded according to their routine to the clinic. As for what should be done after the result, more than $80 \%$ said taking it to the doctor as a determining condition. Conclusion: The descriptions showed that part has knowledge about the screening test, even though it is not possible to accurately quantify the knowledge of each patient. Health professionals play key roles for health information purposes in general, however, greater attention is still needed in promoting women's health.
\end{abstract}

Keywords: Pap smear, Knowledge, Cervical cancer prevention.

${ }^{1}$ Centro Universitário São Lucas, Porto Velho-Rondônia. *E-mail: josiel.nsilva@gmail.com

2 Universidade Federal de Rondônia - UNIR, Porto Velho - Rondônia. 


\section{RESUMEN}

Objetivo: evaluar el conocimiento de las mujeres sobre la prueba de Papanicolaou en un centro de atención médica para mujeres. Método: El estudio fue descriptivo y exploratorio con un enfoque cuantitativo. Para esto, se aplicó un cuestionario con preguntas generales y específicas, luego de firmar el Término de Consentimiento Libre e Informado, que evaluó, entre otros factores, el perfil socioeconómico, la educación y el nivel de conocimiento sobre el examen. Resultados: 100 mujeres participaron en el estudio, en el que el $40 \%$ de las mujeres dejó en claro la relación entre el propósito / prevención del cáncer cervical. Más de la mitad de las mujeres respondieron que sabían los requisitos para el examen. Aunque el $5 \%$ de las personas dijeron que el intervalo entre dos pruebas depende, el resto respondió de acuerdo a su rutina a la clínica. En cuanto a lo que debe hacerse después del resultado, más del $80 \%$ dijo llevarlo al médico como una condición determinante. Conclusión: Las descripciones mostraron que parte tiene conocimiento sobre la prueba de detección, aunque no es posible cuantificar con precisión el conocimiento de cada paciente. Los profesionales de la salud desempeñan funciones clave para fines de información de salud en general, sin embargo, aún se necesita mayor atención para promover la salud de las mujeres.

Palabras clave: Papanicolaou, Conocimiento, Prevención del cáncer cervical.

\section{INTRODUÇÃO}

O câncer cérvico-uterino (CCU) é uma das neoplasias malignas que mais afeta a população feminina no Brasil. Embora existam campanhas que tratam e estimulam à prevenção e a importância dos exames clínicos frequentes, essa situação emergente torna-se cada vez mais um problema de saúde pública (FERNANDES JV e NARCHI SHL, 2002).

Segundo o Instituto Nacional de Câncer (INCA, 2018), o câncer de colo de útero é o terceiro tumor maligno mais frequente na população feminina, atrás do câncer de mama e colorretal, e a quarta causa de morte de mulheres por câncer no Brasil. Estima-se que em 2013 houve 5.430 mortes decorrentes do CCU e em 2016 esperava-se 16.340 novos casos (LOPES VAS e RIBEIRO JM, 2019).

O elevado número da morbimortalidade coloca em evidência o papel do sistema público de saúde, sendo o exame de Papanicolaou o método mais indicado até hoje para o rastreio e detecção de neoplasia. Além de ser acessível e gratuito, esse instrumento tem papel significativo, pois consegue alcançar, e até mesmo regredir complicações do colo de útero em mulheres (PELLOSO SM, et al., 2004).

Esse exame configura-se como a principal estratégia de detecção precoce antes da aparição de sintomas como: sangramento vaginal, corrimento e dor, podendo ser feito nas unidades de saúde da rede pública por profissionais capacitados (IGLESIAS GA, et al., 2019). Ressalta-se a importância de observar alguns fatores de risco durante as consultas como a multiparidade, excesso de peso, estrogênio exógeno, nível socioeconômico, mulheres com menos de 35 anos e portadoras de HPV 16 ou 18 no caso do adenocarcinoma cervical (COSTA TM, et al., 2019).

O Brasil é um dos pioneiros na introdução do exame Papanicolau, contudo ainda se nota uma baixa adesão pelas mulheres (PAULA TC, et al., 2019). Esse cenário pode ser contribuído pela demora nas consultas no Sistema Único de Saúde (SUS), a vergonha de muitas mulheres para a realização do exame, o desconhecimento sobre a importância do procedimento são alguns fatores que dificultam a redução das taxas dessa neoplasia principalmente em mulheres de baixa renda (BRENNA SMF, et al., 2001).

Além disso, Mahecha-Gamboa L (2019), cita 7 barreiras relacionadas a acessibilidade ao sistema como fatores que ainda contribuem para diminuir as taxas de cobertura do $\mathrm{CCU}$, sendo elas: administrativas, geográficas, normativas, diminuição da oferta de vagas, cultural, social e econômico.

De acordo com a observação de Fernandes R e Narchi N (2009), a história natural do CCU, mostra que quanto mais cedo tem-se o diagnóstico, mais chances há de cura devido ao seu lento progresso. Conforme o exposto, é necessário estratégias que vão desde a reformulação da estrutura e acesso a saúde, bem 
como a sensibilização de mulheres acerca dos benefícios da realização do exame Papanicolau. Assim, este estudo tem como finalidade avaliar o conhecimento de mulheres sobre o exame de Papanicolaou em um Centro de Atenção à Saúde da Mulher.

\section{MÉTODOS}

A pesquisa ocorreu em Rondônia, em um ambulatório ginecológico pertencente à instituição privada de ensino superior que destina os atendimentos a mulheres usuárias do Sistema Único de Saúde (SUS), que compõem a população do bairro localizado no centro da cidade e bairros adjacentes. Os critérios de inclusão da pesquisa consideraram mulheres maiores de 18 anos que buscaram atendimento por variadas queixas ginecológicas. As usuárias que não se enquadravam nos critérios foram automaticamente excluídas da pesquisa.

A população feminina do estudo consistiu em 160 mulheres, no entanto apenas 100 estavam dentro dos critérios de inclusão. A coleta foi realizada entre os dias 14 a 28 de agosto de 2018 de segunda-feira à sexta-feira, no período compreendido entre 7:30 às 11:00 horas. As determinações dos critérios avaliativos foram baseadas a partir da estrutura orientada pelo Arco de Maguerez, descrito pelo autor Colombo AA e Berbel NAN (2007), que consiste inicialmente pela observação da realidade a fim de definir uma problemática a ser estudada, seguido pela reflexão e determinação dos pontos observados, teorização auxiliando na construção de respostas para o problema, finalizando com a hipótese de solução que permite a criação de alternativas para auxiliar na resolução do problema levantado.

Os dados coletados foram registrados em um banco de dados do programa Microsoft Excel 2013 e os resultados apresentados de modo descritivo no Microsoft Word 2013 analisando a frequência percentual de cada resultado. Este trabalho foi aprovado pelo Comitê de Ética e Pesquisa sob o número do CAAE 91153218.8.0000.0013, número do parecer 2.799.763 e com a anuência dos participantes por meio do termo de consentimento livre e esclarecido (TCLE).

\section{RESULTADOS}

Inicialmente foi observada a realidade do ambulatório a fim de determinar os problemas existentes, por meio do Arco de Maguerez. Diante disso, foi observado dúvidas das pacientes quanto ao exame Papanicolau, o que determinou os pontos a serem questionados às usuárias.

Para avaliação foi usado um questionário que avaliava a faixa etária, escolaridade, profissão/ocupação, renda familiar, qual a finalidade do exame Papanicolau, importância de fazê-lo, requisitos para realização do exame, periodicidade da realização do exame e condutas após o resultado.

O estudo contou com uma população de 100 pacientes, sendo a faixa etária com média de 39 anos, a idade mínima foi de 18 anos e a máxima de 60 anos. Em relação à escolaridade, $60 \%$ delas tinham o ensino médio completo, $25 \%$ tinham nível superior, já nas opções de ensino fundamental e sem escolaridade, foram $12 \%$ e $3 \%$, respectivamente.

No que tange a profissão/ocupação, as respostas foram variadas, assim como algumas pacientes não responderam o item. Nesse contexto, a fim de identificar e enquadrar no mínimo de opções possíveis as profissões para uma melhor comparação futura, optou-se por dividir em "Não colocou", que apresentou em $14 \%$ dos questionários, apenas $1 \%$ da população era aposentada, $10 \%$ eram estudantes, no rol de empregada o dado foi mais que a metade, $52 \%$. Ademais, também se obteve $9 \%$ como desempregadas, e 10\% eram autônomas.

Em relação à renda familiar (salário mínimo de 2018, $\mathrm{R} \$ 954,00$ ), 12\% tinham menos de 1 salário mínimo, $48 \%$ tinha até 1 salário mínimo, 28\% das mulheres tinham de 2 a 3 salários mínimos, $7 \%$ tinha mais de 4 salários mínimos e outros $5 \%$ da população não colocaram a renda familiar.

Em relação à pergunta que avaliava a finalidade do exame Papanicolau obtivemos variadas respostas que estão exemplificadas (Gráfico 1). 
Gráfico 1 - Finalidade do exame de Papanicolau.

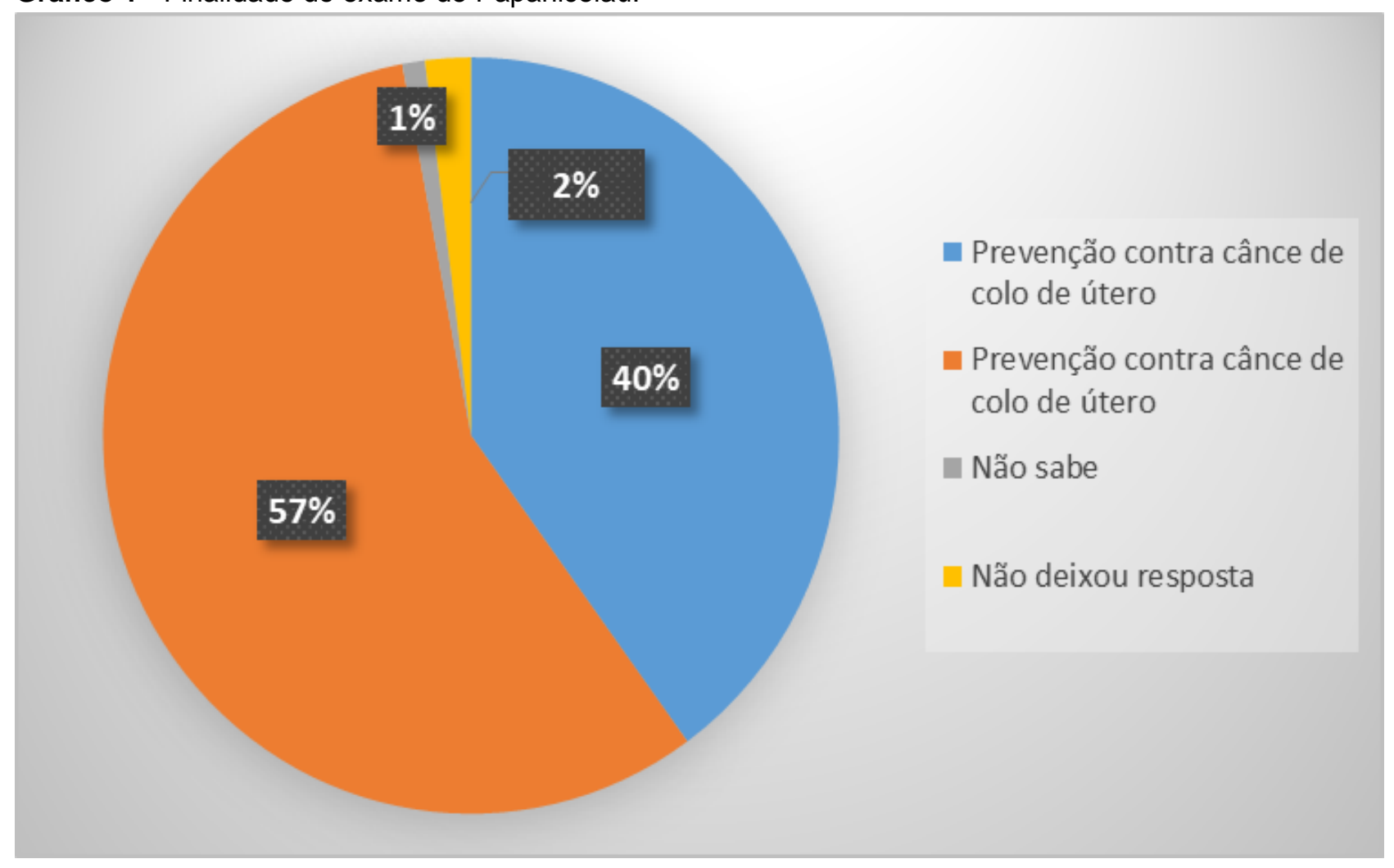

Fonte: Silva JN, et al., 2018.

Nesse primeiro quadro, as respostas variaram de completas para curtas ou até não saber responder. $40 \%$ das pessoas deixaram claro a relação da finalidade com a prevenção do câncer do colo de útero. Apenas uma pessoa (1\%) declarou não saber qual era a finalidade.

Outras duas mulheres (2\%) não responderam, como mostra em NDR. A maioria (57\%) relaciona o exame como mecanismo de prevenção de doenças, exemplo são as respostas curtas escritas "Prevenção" ou só "Grande importância, evitar o câncer". Já ao avaliar a importância da realização do exame obteve-se variadas respostas conforme descrito abaixo (Gráfico 2).

Gráfico 2 - Importância da realização do Papanicolau.

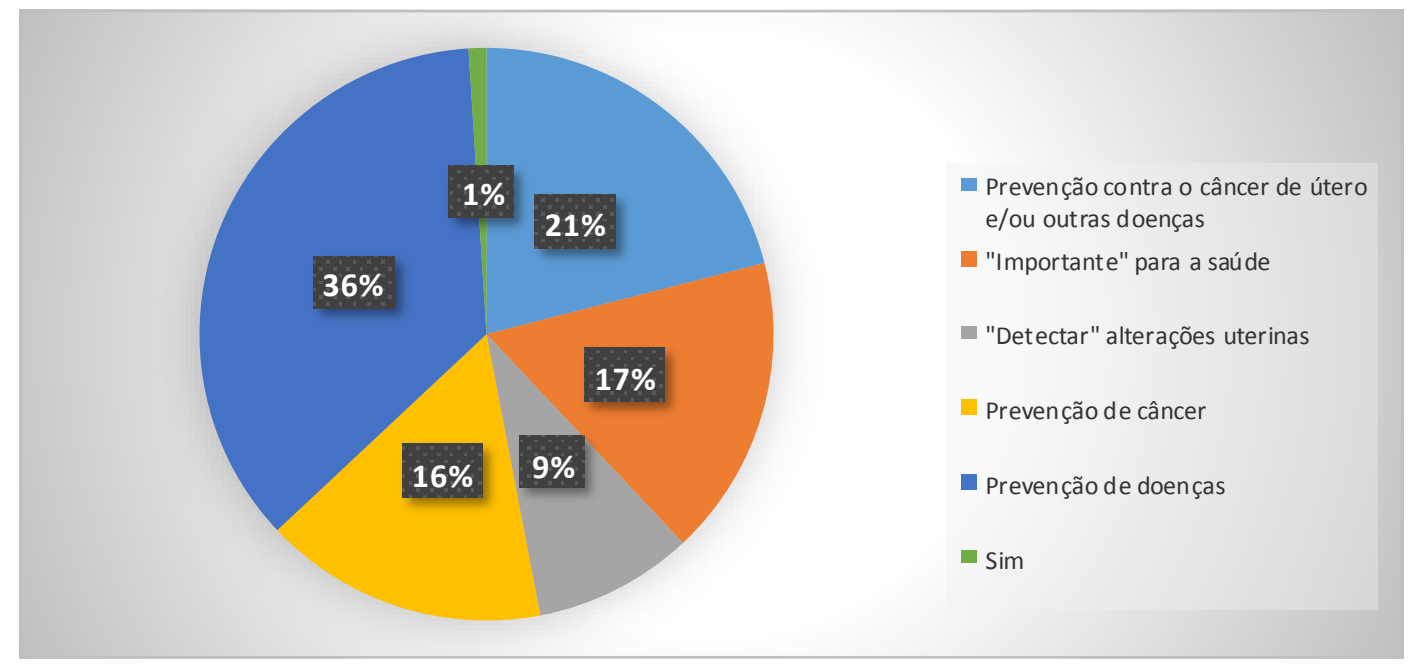

Fonte: Silva JN, et al., 2018. 
Nas respostas deste item, $21 \%$ das mulheres enfatizaram como principal importância da realização do exame a prevenção do câncer cérvico-uterino e/ou outras doenças. A palavra "Importante" aparece em 17\% das respostas; palavras relacionadas ao verbo "detectar" aparecem em $9 \%$ vezes nos questionários, pois grande parte das mulheres já relacionavam preventivo e detecção de alterações na região uterina. A palavra "câncer" aparece em 16\% somente, apesar de um grupo feminino abordar prevenção de doenças, a especificidade sobre o câncer de colo de útero parece ainda ser tímida entre as mulheres. A resposta dada como "Sim" apareceu só uma vez (1\%). Ao levantar os requisitos para a realização do exame, foi questionado se a paciente tinha conhecimento ou não. Os resultados estão descritos abaixo (Gráfico 3).

Gráfico 3 - Respostas das mulheres quanto aos requisitos para a realização do Papanicolau.

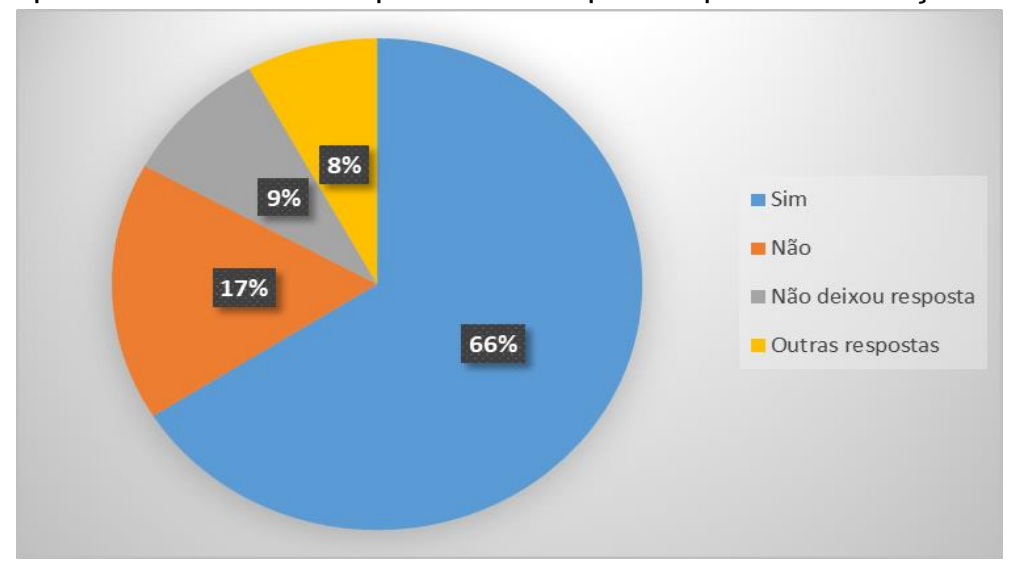

Fonte: Silva JN, et al., 2018.

De acordo com as amostras, quanto a este item, $66 \%$ disseram saber sobre os requisitos do exame preventivo. As respostas que declararam não saber correspondem a $17 \%$. Ainda que o Papanicolaou pareça ser comum entre as mulheres, neste estudo há uma quantidade considerada com desinformação acerca dos requisitos necessários antes do exame ginecológico.

As mulheres que não responderam estão no gráfico expressadas na forma de NDR (não deixaram resposta), e contam com $9 \%$ das respostas. Isso pode mostrar uma incerteza sobre a pergunta que contribui diretamente com o nível de conhecimento das pacientes. Quanto às outras respostas, a maioria respondeu de forma resumida e diferente, não seguindo um mesmo sentido, demonstradas em "Outras respostas" no gráfico com $8 \%$ do total. Pontuou-se também, o conhecimento sobre os intervalos do exame de preventivo das mulheres, com diferentes resultados descritos abaixo (Gráfico 4).

Gráfico 4 - Tempo entre dois exames de Papanicolaou.

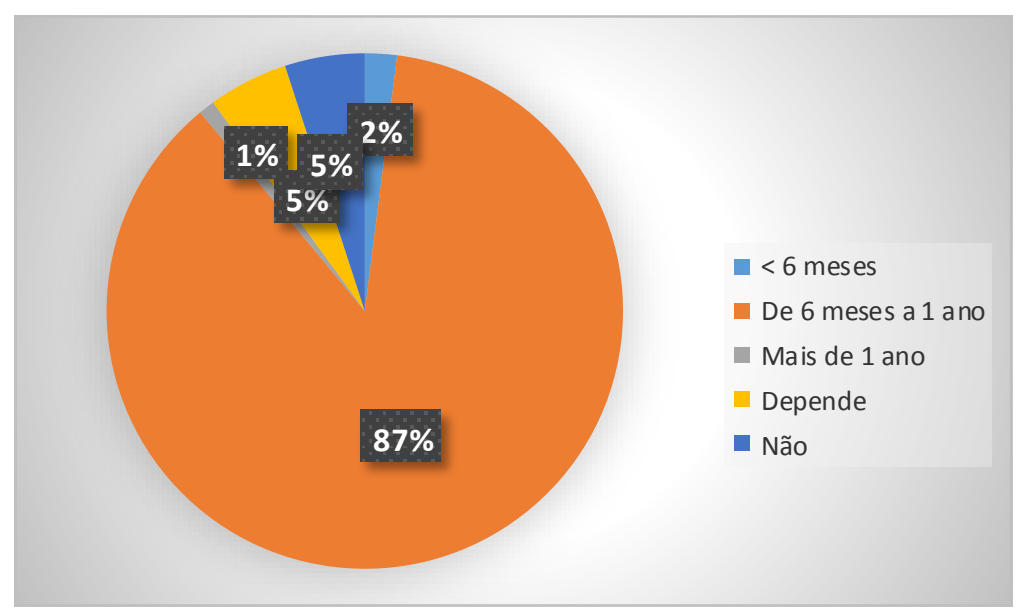

Fonte: Silva JN, et al., 2018. 
As respostas foram bem concordantes umas com as outras, $87 \%$ das mulheres responderam que 0 tempo necessário entre um exame e outro é de 6 meses a 1 ano. Já $5 \%$ disseram não saber sobre o intervalo, outras $5 \%$ disseram ainda que depende do resultado. $2 \%$ das mulheres responderam que era em um tempo curto ou abaixo de 6 meses, e $1 \%$ restante respondeu que era mais de 1 ano o tempo entre dois exames. O conhecimento das mulheres sobre o que deveria ser feito após o recebimento do resultado também foi avaliado, e as respostas estão descritas no (Gráfico 5).

Gráfico 5 - O que deve ser feito após receber o resultado do exame de Papanicolau?

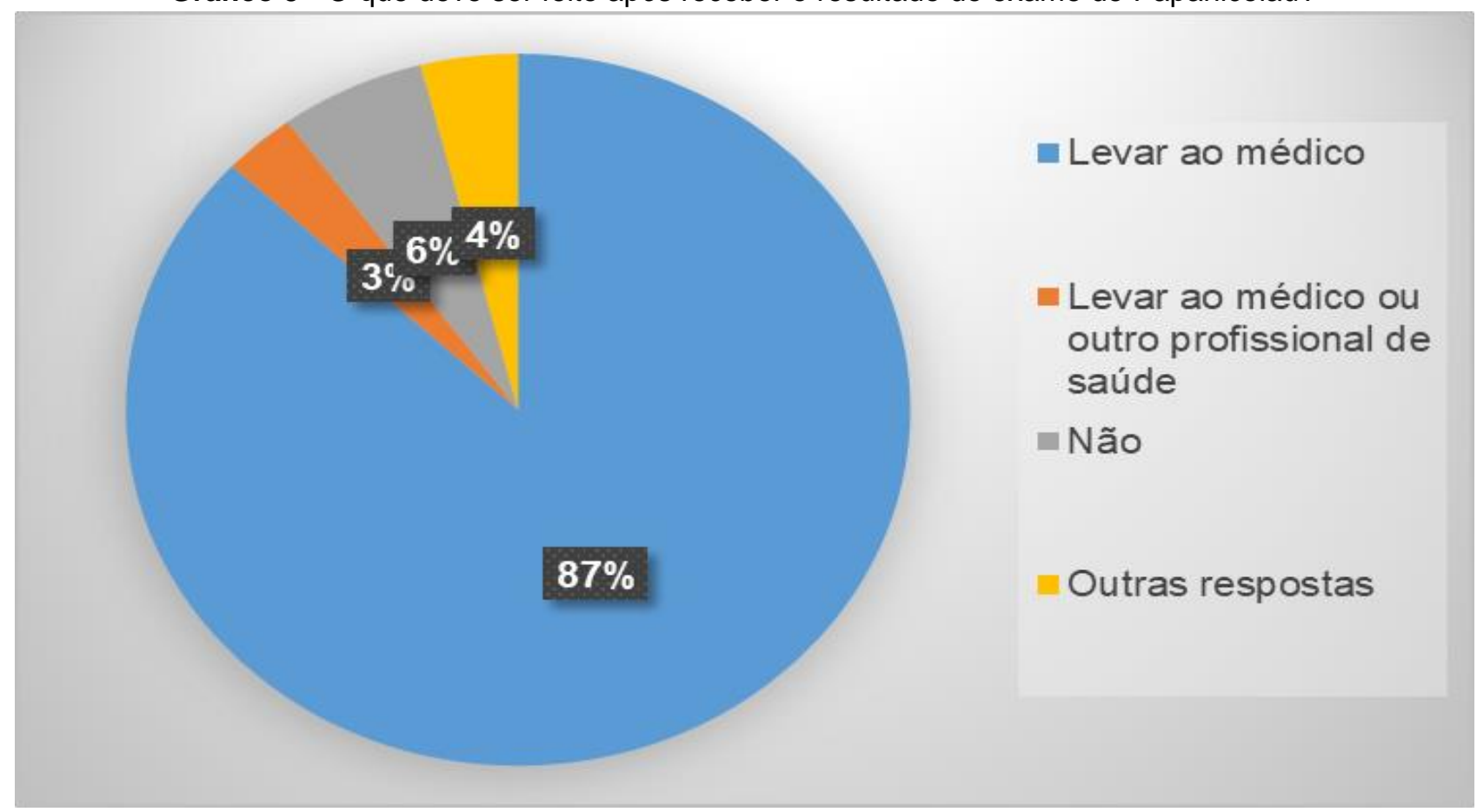

Fonte: Silva JN, et al., 2018.

Nessa análise, $87 \%$ das mulheres foram enfáticas ao dizer "levar ao médico "e/ou responderam "Se der alguma coisa, fazer o tratamento com o ginecologista" como resposta da nona pergunta. Já $6 \%$ delas responderam "Não", 4\% das mulheres responderam de forma não esperada como "Tentar ficar calma e procurar se cuidar" ou "Se cuidar ao máximo" por isso ficaram em "Outras respostas". Os outros $3 \%$ disseram que poderia levar ao médico ou a outro profissional de saúde.

Conforme os resultados obtidos, a busca para compreender os motivos dessa realidade, pode salientar o grau de escolaridade e a pouca informação sobre algumas áreas da temática. Com base nisso, a hipótese de solução foi a estratégia em educação em saúde por meio de materiais educativos impressos.

Por mais que essa estratégia seja considerada deficiente ou com eficácia limitada por ser um modelo unilinear de transmissão de conhecimento, conforme debatido por Teixeira RR (1997), foi necessária a escolha devido o ambulatório não realizar ações de educação em saúde às mulheres atendidas.

O contorno dessa situação irá ocorrer no momento da aplicação da realidade, quando será fornecido a cada equipe do ambulatório os materiais preparados para intervenção e informado a importância de difundir as explicações, a fim de contribuir com a conscientização das pacientes atendidas acerca dos pontos levantados para a pesquisa, exemplificado na Figura 1.

Além disso, será disposto folders com QR Code do material educativo, uma vez que foi observado uso frequente de smartphone, justificando essa alternativa como auxilio na difusão das informações contidas nos panfletos referente a importância do exame, requisitos para realização, tempo entre dois exames e 0 que fazer com o resultado. 
Figura 1 - Fyer informativo e QR code que foram utilizados como intervenção na unidade em que ocorreu a pesquisa.

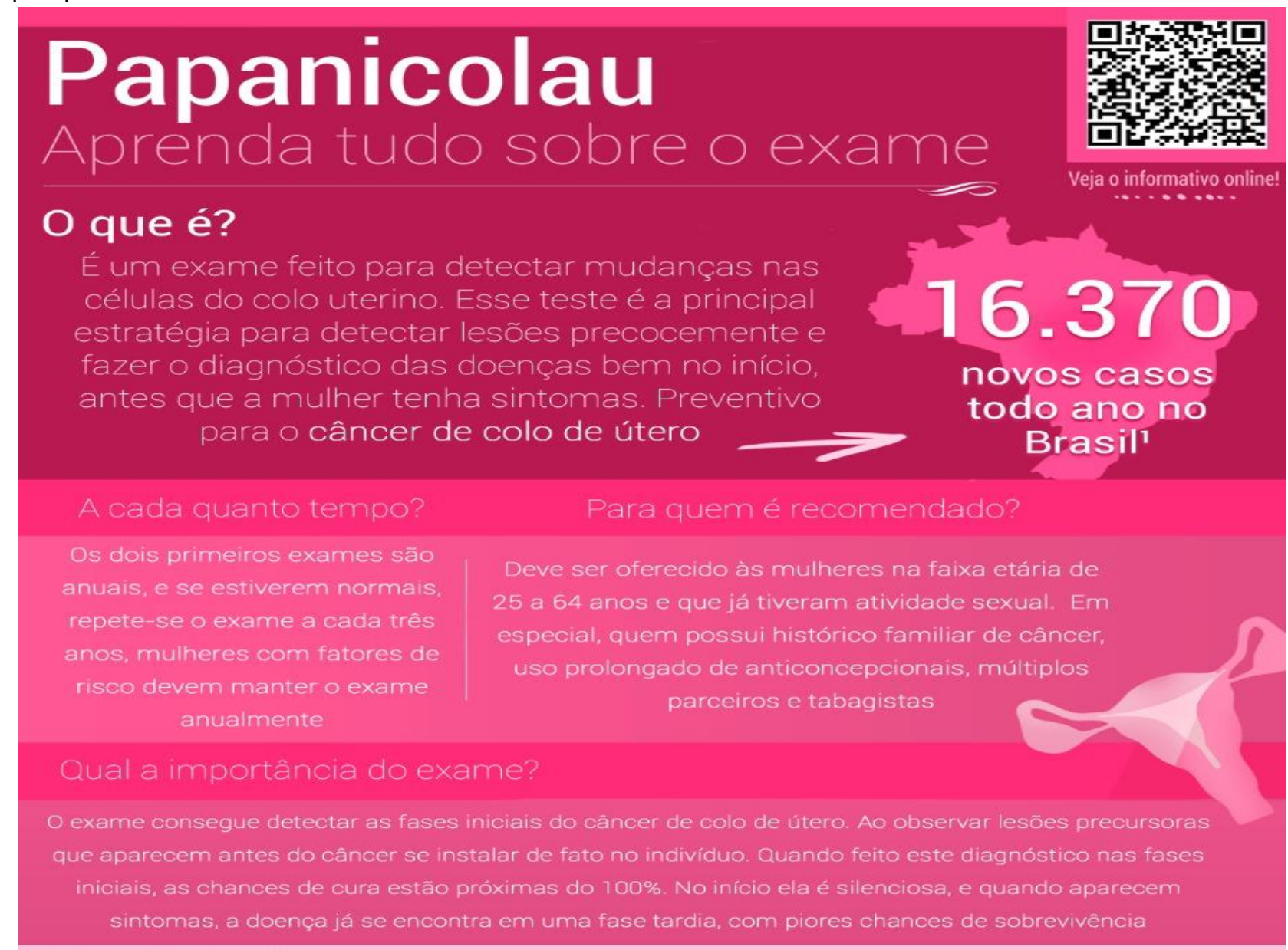

\section{O que fazer após o exame:}

Buscar o resultado e apresentá-lo ao médico. O resultado pode ser.

Amostra insatisfatória - Repetir o exame assim que possivel

- Negativo - Se for seu primeiro negativo, deverá repetir no próximo ano

- Lesão de baixo grau / Infecção pelo HPV - Deverá repetir em seis meses

- Lesão de alto grau - Aguardar a melhor conduta decidida pelo médico

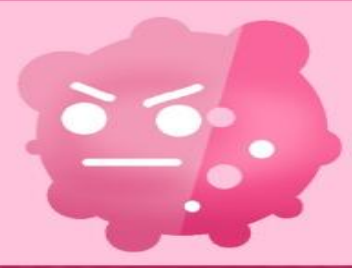

Autores:

Josiel Neves da Silva, Anitha de Cássia Ribeiro da Silva,

Breno de Oliveira Chagas Barreto, Arlindo Gonzaga

Branco Junior Claudete Martins de Lima

Referências:

BRASIL. Instituto Nacional de Câncer José Alencar Gomes da Silva.

Coordenaçăo de Prevençắo e Vigilancia. Divisãó de Detecção Precoce e Apoio à Organizaçăo de Rede. Diretrizes brasileiras para o rastreamento do
câncer do colo do útero. - 2. ed. rev. atual. - Rio de Janeiro: INCA, 2016.

Fonte: Silva JN, et al., 2018.

\section{DISCUSSÃO}

O predomínio das participantes que buscaram atendimento ginecológico estava na faixa etária de 30 anos ou mais. Indo de encontro a esses dados, o estudo desenvolvido por Brenna SMF, et al. (2001) indicou que mulheres mais novas buscam consultas motivadas pelo uso de anticoncepcionais e gravidez. No entanto, no levantamento de dados feitos por Castro LF (2010), indicou que as motivações ocorrem quando há alguma sintomatologia específica de doenças do aparelho reprodutor.

Em relação ao conhecimento, quando questionadas sobre a finalidade do Papanicolaou, $40 \%$ da população estudada somente respondem atrelando prevenção e câncer do colo de útero, como mostra em uma das amostras, "Pra prevenir doenças no colo do útero entre outras doenças como câncer do colo do útero". Também houve resposta como "Descobrir infecção para poder tratar", ou apenas "Se cuidar melhor 
da saúde". Dificuldade em descrever a forma do cuidado é observada, como em "Cuidados apenas cuidados". Já, a fim de justificar que é importante, mas sem prolongamentos, em algumas amostras foram observadas, como "Muito importante", outras quiseram reduzir a finalidade para apenas "Prevenção".

Isso constata que mesmo sendo um exame de rotina para as mulheres, algumas sentem insegurança por demonstrarem pouco conhecimento. Ao contrário dos resultados mostrado por Fernandes $\mathrm{R}$ e Narchi $\mathrm{N}$ (2002) sobre o conhecimento de gestantes de uma comunidade carente, em que $92 \%$ das mulheres afirmaram conhecer e correlacionaram corretamente a finalidade do exame. No entanto, Chiconela FV e Chidassicua JB (2017), apresentou que a maioria das participantes não sabiam relatar a finalidade do CCU, indo ao encontro dos dados da presente pesquisa.

O número de mulheres nesta pesquisa que conheciam a finalidade do exame mostrou-se relevante ao comparar-se com outras. Isso porque, a desinformação e o conhecimento insuficiente que as tem sobre 0 exame revelam a barreira principal para aderir a prevenção de complicações no colo do útero. Em um estudo sobre barreiras da realização do Papanicolau sob a perspectivas da Equipe de Saúde da Família, grande parte das pacientes atendidas desconhecem a finalidade do exame preventivo. As falas das profissionais relatando que as usuárias costuma relacionar prevenção de DST, ou que o exame é para detectar alguma coisa no útero, sem conclusão adequada, corroboram a incerteza e conhecimento sobre 0 Papanicolau (AGUILAR RP e SOARES DA, 2015).

Quando questionadas sobre a importância da realização do exame, algumas respondem pelo viés sentimental, como em "Pra que não venha ocorrer nenhuma coisa grave. Por isso preciso realizar o exame", "Estar sempre informada sobre minha própria saúde e caso ocorra algo poder tratar e quanto antes com mais chance de cura", "Zelar pelo meu bem-estar e prevenir doenças que possam ser tratadas quando detectadas a tempo" e até mesmo "Porque se der alguma coisa, dá tempo de reverter. O estágio avançado é perigoso". Quando a importância foi atrelada com o medo, houve uma amostra que respondeu "Se prevenir, na minha família já teve 3 casos". Mesmo tendo somente $21 \%$ de mulheres demonstrando a importância do Papanicolaou como forma de prevenção do câncer do colo do útero e outras patologias, outras acreditam que a realização do exame está centrada somente em prevenção.

Embora este estudo não pudesse quantificar as igualdades de respostas, apenas similaridades, uma pesquisa semelhante sobre o conhecimento das mulheres do câncer cérvico-uterino de Pelloso SM et al. (2004) mostra-se concordante a esta ao apresentar que as mulheres fazem o exame por reconhecer sua importância, em contrapartida, revelam o medo e a situação-pressão pelos seus resultados. Outro estudo elaborado por Silveira NSP, et al. (2016), também indicou que 40,4\% das mulheres do estudo compreendem a importância do exame para detectar alguma patologia.

Entre as ações que são realizadas na Atenção Básica, destaca-se a disponibilização do exame de Papanicolau. A necessidade de rastreio, controle, promoção de saúde e diagnóstico são metas que o serviço primário à saúde deve desenvolver (BRASIL, 2013). A importância do exame sob a visão de algumas mulheres nesta análise, contudo, demonstrou-se exíguo.

No estudo sobre atitude e prática sobre o exame de prevenção do câncer cervico-uterino de Melo EMF, et al. (2018) mostrou que mais de $64 \%$ das mulheres tinham conhecimento inadequado acerca do exame. Essa situação é capaz de descrever que o desconhecimento, ou o valor dado ao exame de prevenção do CCU pelas mulheres não se restringe somente a uma região territorial.

Logo, a necessidade de ações que estimulam, bem como busque orientá-las sobre o exame torna-se urgente. Uma revisão sistemática sobre intervenções que favorecem a adesão no exame colpocitologia oncótica de Soares MBO e Silva SR (2016), revelou estratégias significativas de vários estudos a fim de instigar a importância do exame de Papanicolaou entre as mulheres.

As atividades educativas sem dúvida influenciam positivamente na mudança do comportamento do público feminino. Nesse sentido, essas foram as escolhidas neste estudo tanto pela facilidade de realizá-las, quanto pela repercussão que poderia tê-las quando integrado a uma ferramenta online. Ademais, é imperioso reiterar nas consultas de rotinas o conhecimento sobre o CCU e ou complicações, pois ainda que 
as mulheres compareçam em seus exames periódicos, a existência de lacunas sobre o exame ou sobre sua saúde que não foram anteriormente sanadas podem persistir.Quando questionadas sobre os requisitos do exame, respostas com o "Sim" aparecem em $66 \%$ dos questionários, ainda que respondam de forma geral, não é possível analisar o nível de conhecimento, pois as interpretações podem ser diversificadas.

O "Não" aparece em 17\% das amostras, e "não deixaram respostas" (NDR) aponta em 9\%, seja por dúvida seja por embaraço em responder de modo conclusivo. No entanto, algumas respostas mostraram-se coerentes, porém incompletas como em "Mulheres sexualmente ativas". Houve também conclusões no sentido de "Não ter relações sexuais pelo menos três dias antes e após a menstruação" e até incertas como "Uma vez que eu fiz tive que tomar banho, outra vez não". No questionamento quanto ao tempo mínimo entre dois exames, muitas mulheres responderam de acordo com as suas rotinas à coleta, a maioria (87\%) colocou de 6 meses a 1 ano. As que disseram que é menos de 6 meses e mais de 1 ano foram $2 \%$ e $1 \%$, respectivamente.

Outros $" 5 \%$ responderam que dependia do resultado. Somente $5 \%$ disseram não saber. A prevalência desse estudo é semelhante ao desenvolvido por Borges MFSO, et al. (2012), já que $61,7 \%$ das participantes informaram realizar o exame com uma periodicidade anual. Mesmo assim, os conhecimentos em torno da periodicidade respondem às expectativas, uma vez que é primordial tê-la como um aliado para a saúde feminina. De acordo com o INCA (2018), a realização do exame em mulheres que estão entre 25 e 60 anos de idade após dois resultados normais, pode ser feita a cada três anos. Porém, as respostas desta questão evidenciam as orientações recebidas pelas mulheres dos profissionais de saúde com os quais elas frequentam, e estão em consonância com seu ritmo ao consultório médico.

No que tange ao questionamento sobre o que deve ser feito após receber o resultado, somente $6 \%$ declarou não saber, e outros $4 \%$ desviaram-se do propósito da resposta, como em "Se manter sempre higienizada, tomar ou passar pomada sempre que necessário". $87 \%$ das amostras foram diretas ao correlacionar a tríade cuidado, saúde e a necessidade do médico para fim de diagnóstico. Outros $3 \%$ demonstraram levar ao médico ou outro profissional de saúde. Esse dado é similar ao estudo mostrado Valente CA, et al. (2009), pois $91 \%$ da amostra tinha a informação de que deveriam buscar o resultado e levá-lo a um profissional de saúde. A esse respeito, o resultado demonstra que mesmo que um grupo feminino não tenha domínio para algumas perguntas, o médico é o principal profissional em que pode recorrer depois do recebimento dos resultados.

\section{CONCLUSÃO}

Nesta pesquisa, percebeu-se que há uma parcela grande com informações inconclusivas ou até mesmo não sabendo responder de forma esperada respostas relacionadas ao exame de rastreio Papanicolaou. Nessa perspectiva, também se correlacionam a prevenção de doenças mesmo demonstrando ter pouco conhecimento ao falar do câncer no corpo do útero. Desse modo, esse reflexo de conhecimento mostra ser não só um problema que envolve somente a mulher ou sua atitude, mas muito mais que isso, os programas de promoção de saúde de países subdesenvolvidos, como do Brasil. Assim, as medidas em educação em saúde e a participação dos profissionais de saúde são necessárias na função de divulgação da informação, pois assume papel preponderantes para a mudança do cenário atual.

\section{AGRADECIMENTOS E FINANCIAMENTO}

Agradecemos a permissão da coleta de dados no ambulatório, aos profissionais, participantes, auxiliares da pesquisa e orientadores.

\section{REFERÊNCIAS}

1. AGUILAR RP, SOARES DA. Barreiras à realização do exame Papanicolau: perspectivas de usuárias e profissionais da Estratégia de Saúde da Família da cidade de Vitória da Conquista-BA. Revista de Saúde Coletiva, Rio de Janeiro, 25 [ 2 ]: 359-379, 2015. 
2. BRASIL. Instituto Nacional de Câncer José Alencar Gomes da Silva. Coordenação de Prevenção e Vigilância. Divisão de Detecção Precoce e Apoio à Organização de Rede. Diretrizes brasileiras para o rastreamento do câncer do colo do útero.- 2 ${ }^{a}$. ed. rev. atual. - Rio de Janeiro: INCA, 2016.

3. BRASIL. Ministério da Saúde. Secretaria de Atenção à Saúde. Departamento de Atenção Básica. Controle dos Cânceres do Colo do Útero e Mama. CADERNOS DE ATENÇÃO BÁSICA. Brasília: Ed. Ministério da Saúde, 2013. páginas 17-18.

4. Brenna, S.M.F.; Hardy, E.; Zeferino, L.C.; Namura, I. Conhecimento, Atitude E Prática Do Exame De Papanicolaou Em Mulheres Com Câncer De Colo Uterino. Cad. Saúde Pública [Online]. 2001, Vol.17, N.4, Pp.909-914. Issn 0102311x. Http://Dx.Doi.Org/10.1590/S0102-311x2001000400024.

5. BORGES MFSO et al. Prevalência Do Exame Preventivo De Câncer Do Colo Do Útero Em Rio Branco, Acre, Brasil, E Fatores Associados À Não-Realização Do Exame. Cad. Saúde Públic, [F.L.], V. 28, N. 6, P.1156-1166, Set. 2017.

6. CASTRO LF. Exame Papanicolaou:O Conhecimento Das Mulheres Sobre O Preventivo E A Estratégia Do Psf No Combate Ao Câncer De Colo De Útero. 2010. 20 F. Tcc (Graduação) - Curso De Curso De Especialização Em Atenção Básica Em Saúde Da Família, Universidade Federal De Minas Gerais, Minas Gerais, 2010.

7. CHICONELA FV, CHIDASSICUA JB. Conhecimentos E Atitudes Das Mulheres Em Relação Ao Exame Preventivo Do Câncer Do Colo Uterino. Rev. Eletr. Enf. [Internet]. 2017.

8. COSTA TM. 651artigos Originaispapilomavírus Humano E Fatores De Risco Para Adenocarcinoma Cervical Noestado De Pernambuco, Brasil. Rev. Bras. Saúde Mater. Infant., [F.L.], V. 19, N. 3, P.651-660, Jun. 2019.

9. FERNANDES JV. Conhecimentos, Atitudes E Prática Do Exame De Papanicolaou Por Mulheres, Nordeste Do Brasil. Rev. Saúde Pública [Online]. 2009, Vol.43, N.5, Pp.851-858. Epub Sep 18, 2009. Issn 0034-8910. Http://Dx.Doi.Org/10.1590/S0034-89102009005000055.

10. FERNANDES R, NARCHI N. Conhecimento De Gestantes De Uma Comunidade Carente Sobre Os Exames De Detecção Precoce Do Câncer Cérvico-Uterino E De Mama, 2001.

11. IGLESIAS GA et al. Conhecimento E Adesão Ao Papanicolau De Mulheres De Uma Rede De Atenção Primária À Saúde. Rev Ciênc Med. 2019;28(1):21-30. Http://Dx.Doi.Org/10.24220/2318-0897v28n1a4008.

12. INSTITUTO NACIONAL DO CÂNCER (INCA). Câncer Do Colo Do Útero. Rio De Janeiro, 2018. Disponível Em: Http:Https:/Www.Inca.Gov.Br/Tipos-De-Cancer/Cancer-Do-Colo-Do-Utero. Acesso Em: 21 De Jan. 2018.

13. LOPES VAS, RIBEIRO JM. Fatores Limitadores E Facilitadores Para O Controle Do Câncer De Colo De Útero: Uma Revisão De Literatura. Ciência \& Saúde Coletiva, [F.L.], V. 24, N. 9, P.3431-3442, Fev. 2018.

14. MAHECHA-GAMBOA L et al. Conducta Frente A La Prueba De Papanicolaou: La Voz De Las Pacientes Ante La Neoplasia De Cuello Uterino. Revista Colombiana De Enfermería. 2019, V. 18, N. 1, E002.

15. MELO EMF, et al. Câncer cervico-uterino: conhecimento, atitude e prática sobre o exame de prevenção. Rev. Bras. Enferm. vol.72 supl.3 Brasília dez. 2019 Epub 13-Dez-2019.

16. PELLOSO SM et al. Conhecimento Das Mulheres Sobre O Câncer Cérvico-Uterino, 2004. Disponível Em:<Https://Www.Nescon.Medicina.Ufmg.Br/Biblioteca/Imagem/0261.Pdf>. Acesso em: 30 De Maio 2018.

17. PAULA, TC et al. Detecção Precoce E Prevenção Do Câncer De Colo Uterino: Saberes E Práticas Educativas. Enferm. Foco, [F.L.], V. 10, N. 2, P.47-51, maio 2019.

18. SILVEIRA NSP et al. Cknowledge, Attitude And Practice Of The Smear Test And Its Relation With Female Age. Rev. Latino-Am. Enfermagem. 2016;24:E2699.

19. SOARES MBO, SILVA SR. Intervenções que favorecem a adesão ao exame de colpocitologia oncótica: revisão integrativa. Rev Bras Enferm [Internet]. 2016 mar-abr;69(2):404-14

20. TEIXEIRA RR. Modelos Comunicacionais E Práticas De Saúde. Interface - Comunic., Saude, Educ., V.1, N.1, P.740, 1997.

21. VALENTE CA et al. Conhecimento de mulheres sobre o exame de Papanicolaou. Rev Esc Enferm USP 2009; 43(Esp 2):1193-8 